Research Article

\title{
Multiobjective Intelligent Cooperative Design for the Multilayer Interference Fit
}

\author{
Ke Ning $\left(\mathbb{D},{ }^{1,2}\right.$ Jianmei Wang $\left(\mathbb{D},{ }^{1,2}\right.$ Hongwei Jiang, ${ }^{3}$ Dan Xiang, ${ }^{4}$ and \\ Dingbang $\mathrm{Hou}^{2}$ \\ ${ }^{1}$ Engineering Research Center Heavy Machinery Ministry of Education, Taiyuan University of Science and Technology, \\ Taiyuan, China \\ ${ }^{2}$ School of Mechanical Engineering, Taiyuan University of Science and Technology, Taiyuan, China \\ ${ }^{3}$ Technology Center, Taiyuan Heavy Machinery Group Co., Ltd., Taiyuan, China \\ ${ }^{4}$ Oil-Film Bearing Branch, Taiyuan Heavy Machinery Group Co., Ltd., Taiyuan, China \\ Correspondence should be addressed to Jianmei Wang; wjmhdb@163.com
}

Received 20 June 2019; Revised 9 August 2019; Accepted 23 September 2019; Published 13 October 2019

Academic Editor: Francesco Pellicano

Copyright ( 2019 Ke Ning et al. This is an open access article distributed under the Creative Commons Attribution License, which permits unrestricted use, distribution, and reproduction in any medium, provided the original work is properly cited.

\begin{abstract}
Interference fit has extensively been applied in different mechanical fields due to its advantages such as compactness and high rigidity. With the actual needs of engineering, the multilayer interference fit has also been widely used. It is significant for the design and manufacture of multilayer interference fit to consider stress concentration problem and achieve multiobjective and multivariable collaborative optimization in the optimization algorithm. To achieve this goal, the mechanical model and reliability mathematical model of the multilayer interference fit are established. Giving an evaluation method of reliability, the recommended range of interference amount is obtained. Considering the lightweight design and stress concentration problems, a multiobjective intelligent cooperative design (MOICD) method for the multilayer interference fit is proposed, and multiparameter analysis is done. Taking the typical wind turbine's shrink disk of three-layer interference fit structure as an application example, the key design parameters of the shrink disk are determined, and its sample data are obtained using the orthogonal experimental method. Considering yield strength, torque, and mass, the approximate mathematical model of response surface using the Kriging algorithm is given. Nondominated sorting genetic algorithm-II (NSGA-II) is selected as the optimal algorithm to realize MOICD of the shrink disk. Numerical analysis and analytical calculation proved that the optimization index of the MOICD method is improved compared with the traditional method. The sample is tested on a specific test bench, and the test results meet the design requirements, which verifies the feasibility of MOICD.
\end{abstract}

\section{Introduction}

Interference fit has advantages such as precision positioning and high load-carrying capacity, comparing with key connection and pin connection. It has widely been used in heavy machinery, new energy sources, and general machinery, such as semipermanently connected gears, wheels, flanges, and similar mechanical components [1].

Many studies on the interference fit have been performed over the years. Sun et al. [2] researched the process of a pin interference fit and the subsequent fatigue damage evolution, and the result shows that the effect of the interference fit on the fatigue life improvement is beneficial. $\mathrm{Wu}$ et al. $[3,4]$ deepened the study of the interference fit to improve fatigue life and used the spring-mass model to explore the fatigue enhancing mechanism of interference. The study of Zeng et al. [4] on an interference fit between hole and rivet shows that an interference fit can not only improve fatigue life but also enhance strength effect by changing size. Bahloul et al. [5] used the interference fit 


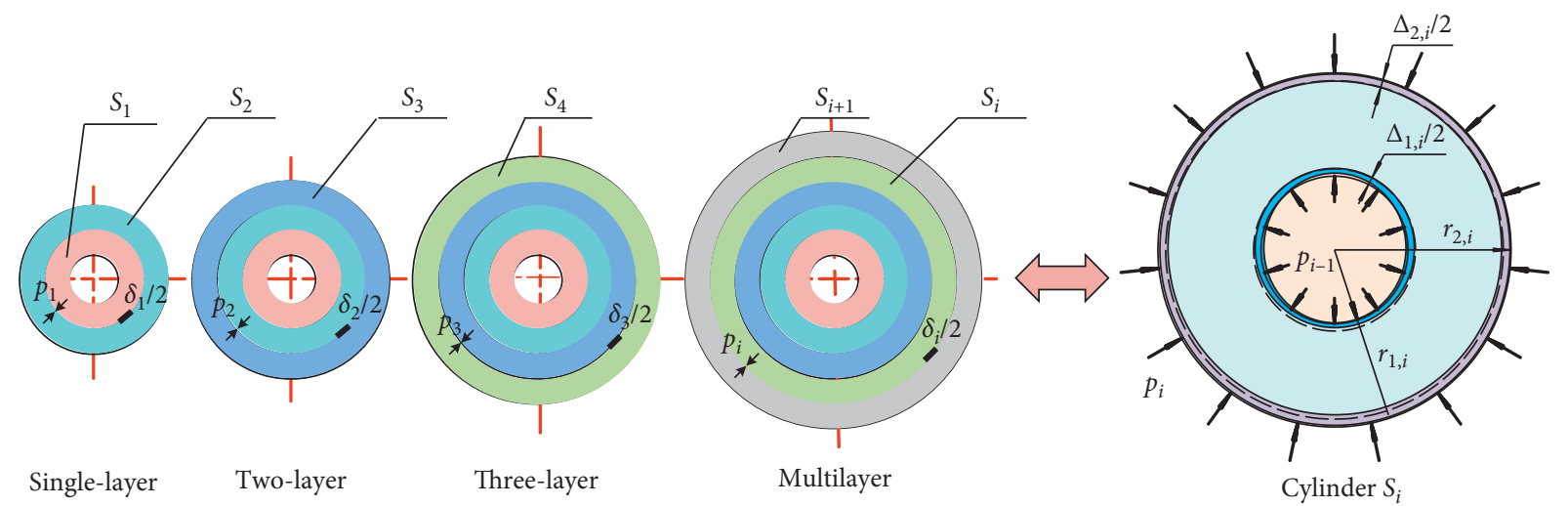

Figure 1: The diagram of a multilayer interference fit.

process as a technique to arrest existing crack in mechanical structures, improving the fatigue life and investigating the effects of different hole diameters and sizes to optimize the interference fit process parameters. Zou et al. [6] established the general mechanical model during the interference fit pin installation process, predicting the delamination onset at different interference fit percentages, the insertion load, and stress. Strozzi et al. [1] addressed the stress concentrations developed from the static press-fit of the shaft-hub interference fit, and various design charts have been compiled that report the elastic stress concentrations within the hub versus the proposed normalizing parameter, including fillet radius, shaft radius, and interference. Wang et al. [7] deduced the stress distribution equation of the interference fit based on the stress concentration problem, and a new theoretical model of stress distribution prediction was established. Most of the above research was aimed at single-layer interference fit, but with the actual needs of engineering, many use double or multilayer interference fit structure to achieve load transfer. Wang et al. [8-10] deduced the mechanical model of the multilayer interference fit, analyzed its bearing capacity by the finite element method, and proposed a reliable and robust design method. Qiu and Zhou [11] investigated the temperature effect of the multilayer interference fit. Bai et al. [12] established a theoretical model of the multilayer interference fit considering the effect of centrifugal force. You et al. [13, 14] developed an analytical solution for the mechanical behaviors associated with a three-dimensional anisotropic multilayered medium under moving loads, and the dynamic response analysis of multilayer structures was realized. However, until now, there has been little research on the design algorithm of the multilayer interference fit considering stress concentration problem, as well as little research on optimization design based on multiobjective and multivariable interaction.

To solve the above problem, this work gives the reliability mathematical model of the multilayer interference fit based on the mechanical model. The recommended range of interference is determined. Considering the lightweight design and stress concentration problems, the multiobjective and multivariable synergistic relationship is established, and the multiobjective intelligent cooperative design (MOICD) method for the multilayer interference fit is proposed. Constructing an approximate mathematical model of the response surface, the prediction of load transfer, material yield strength, and equipment weight is realized. Nondominated sorting genetic algorithm-II (NSGA-II) is selected as the optimal algorithm to optimize the multiobjective model composed of the response surface mathematical model. The typical three-layer interference fit of the wind turbine's shrink disk is taken as an example to design. Based on finite element analysis and mechanical model calculation, the optimized shrink disk is validated to meet the requirements of optimization, and the sample is verified by a special test bench, which meets the operational requirements.

\section{Reliability Model of Multilayer Interference Fit}

2.1. Theoretical Deduction. According to the thick-walled cylinder theory, the multilayer interference fit can be simplified as a multilayer cylinder, as shown in Figure 1, and the cylinder is numbered $S_{1}, \ldots, S_{i+1}$ from inside to outside. $p_{i}$ is defined as the contact pressure of the interference layer between $S_{i}$ and $S_{i+1}$, and the interference amount between $S_{i}$ and $S_{i+1}$ is $\delta_{i}$.

The matrix expression between contact pressure and interference amount of the multilayer interference fit can be derived as

$$
M P=\delta
$$

where $i=1,2, \ldots, n, \delta=\left[\delta_{1}, \delta_{2}, \ldots, \delta_{i}\right]^{T}, \quad P=\left[p_{1}, p_{2}\right.$, $\left.\ldots, p_{i}\right]^{T}, d_{1, i}$ is the inner diameter of $S_{i}, d_{2, i}$ is the outer diameter of $S_{i}$, and $v_{\mathrm{i}}$ and $E_{\mathrm{i}}$ are Poisson's ratio and modulus of elasticity of $S_{i}$, respectively. 


$$
\begin{aligned}
K_{1, i} & =\frac{\left[\left(1-v_{i}\right)+\left(1+v_{i}\right) n_{i}^{2}\right] d_{1, i}}{E_{i}\left(n_{i}^{2}-1\right)}, \\
K_{2, i} & =\frac{2 d_{1, i} n_{i}^{2}}{E_{i}\left(n_{i}^{2}-1\right)}, \\
K_{3, i} & =\frac{2 d_{2, i}}{E_{i}\left(n_{i}^{2}-1\right)}, \\
K_{4, i} & =\frac{\left[\left(1+v_{i}\right)+\left(1-v_{i}\right) n_{i}^{2}\right] d_{2, i}}{E_{i}\left(n_{i}^{2}-1\right)}, \\
n_{i} & =\frac{d_{2, i}}{d_{1, i}},
\end{aligned}
$$$$
M=\left[\begin{array}{ccccccc}
\left(K_{1,2}+K_{4,1}\right) & -K_{2,2} & 0 & \cdots & 0 & 0 & 0 \\
-K_{3,2} & \left(K_{1,3}+K_{4,2}\right) & -K_{2,3} & 0 & \cdots & 0 & 0 \\
0 & -K_{3,3} & \left(K_{1,4}+K_{4,3}\right) & -K_{2,4} & 0 & \ldots & 0 \\
\vdots & & \vdots & \vdots & \vdots & & \vdots \\
0 & \ldots & 0 & -K_{3, n-2} & \left(K_{1, n-1}+K_{4, n-2}\right) & -K_{2, n-1} & 0 \\
0 & 0 & \ldots & 0 & -K_{3, n-1} & \left(K_{1, n}+K_{4, n-1}\right) & -K_{2, n} \\
0 & 0 & 0 & \cdots & 0 & -K_{3, n} & \left(K_{1, n+1}+K_{4, n}\right)
\end{array}\right] .
$$

The boundary conditions of the multilayer interference fit with clearance fit can be expressed as follows:

$$
\left\{\begin{array}{l}
\Delta_{2,1}-\Delta_{1,2}=\Delta_{1} \\
\Delta_{2,2}-\Delta_{1,3}=\Delta_{2} \\
\Delta_{2, i-1}-\Delta_{1, i}=\Delta_{i-1} \\
\Delta_{1, i+1}-\Delta_{2, i}=\delta_{i}
\end{array}\right.
$$

where $\Delta_{1, i}$ and $\Delta_{2, i}$ are the radial displacements of inner and outer surfaces after compression of $S_{i} \cdot \Delta_{1, i}=K_{1, i} p_{i-1}-K_{2, i} p_{i}$ and $\Delta_{2, i}=K_{3, i} p_{i-1}-K_{4, i} p_{i} ; \Delta_{1}, \Delta_{2}, \ldots, \Delta_{i-1}$ are the assembly clearance between $S_{1}$ and $S_{2}, S_{2}$ and $S_{3}, \ldots, S_{i-1}$ and $S_{i}$.

According to equations (1) and (3), the contact pressure $p_{1}$ of first-layer interference is expressed as

$$
p_{1}=N_{1} \cdot \Delta_{1}+N_{2} \cdot \Delta_{2}+\cdots+N_{i-1} \cdot \Delta_{i-1}+N_{i} \cdot \delta_{i},
$$

where $i=1,2, \ldots, n$ and $N_{1}, \ldots, N_{i}$ is the relational expression among $K_{1, i}, \ldots, K_{4, i} \cdot[15]$.

Taking the three-layer interference fit as an example, some formulas of equation (4) are as follows:

$$
\left\{\begin{array}{l}
N_{1}=-\left[\left(K_{1,4}+K_{4,3}\right)\left(K_{4,2}+K_{1,3}\right)-K_{2,3} K_{3,3}\right] \div\left[K_{2,3} K_{3,3}\left(-K_{4,1}-K_{1,2}\right)-\left(K_{1,4}+K_{4,3}\right)\right] \div\left[K_{3,2} K_{2,2}+\left(K_{4,2}+K_{1,3}\right)\left(-K_{4,1}-K_{1,2}\right)\right], \\
N_{2}=-K_{2,2}\left(K_{1,4}+K_{4,3}\right) \div\left[K_{2,3} K_{3,3}\left(-K_{4,1}-K_{1,2}\right)-\left(K_{1,4}+K_{4,3}\right)\right] \div\left[K_{3,2} K_{2,2}+\left(K_{4,2}+K_{1,3}\right)\left(-K_{4,1}-K_{1,2}\right)\right], \\
N_{3}=K_{2,3} K_{2,2} \div\left[K_{2,3} K_{3,3}\left(-K_{4,1}-K_{1,2}\right)-\left(K_{1,4}+K_{4,3}\right)\right] \div\left[K_{3,2} K_{2,2}+\left(K_{4,2}+K_{1,3}\right)\left(-K_{4,1}-K_{1,2}\right)\right] .
\end{array}\right.
$$

Combining equations (3) and (4), the contact pressure of other interference layers can be deduced as

$$
p_{i}=G_{i}-H_{i} p_{i-2}+J_{i} p_{i-1}
$$

where $i \geq 2$ and $G, \ldots, J$ is certain coefficient related to boundary conditions, which satisfies $G_{i}=\left(\Delta_{i-1} / K_{2, i}\right)$, $H_{i}=\left(K_{3, i-1} / K_{2, i}\right)$, and $J_{i}=\left(\left(K_{4, i-1}+K_{1, i}\right) / K_{2, i}\right)$. 
2.2. Mechanical Model. Considering that the primary function of the multilayer interference fit is to transfer torque, the mechanical model is constructed. The first-layer interference, i.e., the contact surface between $S_{1}$ and $S_{2}$, meets the rated torque transfer. The minimum design contact pressure $p_{1, d \text { min }}$ is as follows:

$$
p_{1, d \text { min }}=\frac{2 M_{t}}{\pi \mu_{1} d_{1}^{2} l_{1}}=\frac{A}{\mu_{1}},
$$

where $A=\left(2 M_{t} / \pi d_{1}^{2} l_{1}\right), M_{t}$ is the rated transfer torque, $\mu_{1}$ is the friction coefficient between $S_{1}$ and $S_{2}, d_{1}$ and $l_{1}$ are the combined diameter and contact length of first-layer interference, respectively.

To ensure the reliability of the multilayer interference fit, the maximum contact pressure of each interference layer should be less than the plastic deformation or fracture limit of the material. Making $S_{1}$ as an enveloped part of first-layer interference, the maximum contact pressure between $S_{1}$ and $S_{2}$ allowed for no plastic deformation or fracture should be as follows.

When cylinder $S_{1}$ is a plastic material,

$$
p_{1, \max 1}=B \cdot \sigma_{S_{1}}
$$

where $B=\left(\left(1-q_{a}^{2}\right) / 2\right), q_{a}=\left(d_{1,1} / d_{1}\right)$, and $\sigma_{S_{1}}$ is the yield strength of $S_{1}$.

When cylinder $S_{1}$ is a brittle material,

$$
p_{1, \max 1}=\frac{B \cdot \sigma_{B 1}}{c},
$$

where $\sigma_{B 1}$ is the tensile strength of $S_{1}$ and $c$ is the safety factor for the strength of brittle material.

The enveloping part of first-layer interference takes $S_{2}, S_{3}, \ldots, S_{i+1}$ as a whole; the maximum contact pressure between $S_{1}$ and $S_{2}$ allowed for no plastic deformation or fracture should be as follows.

When the cylinder $S_{2}, S_{3}, \ldots, S_{i+1}$ is a plastic material,

$$
p_{1, \max 2}=D \cdot \sigma_{S_{2}}^{\prime}
$$

where $D=\left(\left(1-q_{b}^{2}\right) / \sqrt{3+q_{b}^{2}}\right), q_{b}=\left(d_{1} / d_{2, i+1}\right)$, and $\sigma_{S_{2}}^{\prime}$ is the equivalent yield strength of $S_{2}, S_{3}, \ldots, S_{i+1}$.

When the cylinder $S_{2}, S_{3}, \ldots, S_{i+1}$ is a brittle material,

$$
p_{1, \max 2}=\frac{D \cdot \sigma_{B 2}^{\prime}}{c},
$$

where $\sigma_{B 2}^{\prime}$ is the equivalent tensile strength of $S_{2}, S_{3}, \ldots, S_{i+1}$.

The maximum contact pressure between cylinders $S_{1}$ and $S_{2}$ is $p_{1, \max }=\min \left(p_{1, \max 1}, p_{1, \max 2}\right)$, and the mechanical model of the multilayer interference fit can be deduced by combining equations $(7) \sim(11)$ :

$$
\left\{\begin{array}{l}
p_{1, d}>p_{1, d \min } \\
p_{1, d}<p_{1, \max } \\
\ldots \\
p_{i, d}<p_{i, \max }
\end{array}\right.
$$

where $i=1,2, \ldots, n, p_{i, d}$ is the design contact pressure of $i$-th layer interference (cylinder $S_{\mathrm{i}}$ and $S_{\mathrm{i}+1}$ ), $p_{i, \max }$ is the maximum permissible contact pressure, i.e., $p_{i, \max }=$ $\min \left(p_{i, \max 1}, p_{i, \max 2}\right), p_{i, \max 1}$ is the maximum permissible contact pressure for the enveloped part, and $p_{i, \max 2}$ is the maximum permissible contact pressure for the enveloping part.

2.3. Reliability Mathematical Model. According to the classical interference fit design method [16], the basic random variables $X$ include friction coefficient $\mu_{1}, \ldots, \mu_{i}$, assembly clearance $\Delta_{1}, \ldots, \Delta_{i-1}$ of first-layer to $i$-th layer interference, the design interference amount $\delta_{i}$ of $i$-th layer interference, and the yield strength $\sigma_{S_{i}}$ of cylinder $S_{i} ; X=$ $\left[\mu_{1}, \ldots, \mu_{i}, \Delta_{1}, \ldots, \Delta_{i-1}, \delta_{i}, \sigma_{S_{1}}, \ldots, \sigma_{S_{i}}\right]^{T}$. The basic random variables are assumed to obey normal distribution and mutual independence.

The reliability mathematical model of the multilayer interference fit is established based on the mechanical model, and several mechanical conditions must be satisfied at the same time so that the overall reliability can be guaranteed. It has distinct series characteristics. The reliability mathematical model is as follows [8]:

$$
\left\{\begin{array}{l}
R(X)=\prod_{k=0}^{i+1} R_{i}(X), \\
R_{0}(X)=\int_{g_{0}(X)>0} f_{0}(X) \mathrm{d} X, \\
R_{1}(X)=\int_{g_{1}(X)>0} f_{1}(X) \mathrm{d} X, \\
\cdots \\
R_{i}(X)=\int_{g_{i}(X)>0} f_{i}(X) \mathrm{d} X,
\end{array}\right.
$$

where $g_{0}(X), g_{1}(X), \ldots, g_{i}(X)$ are the corresponding state functions, $R_{0}(X)$ is the reliability index to satisfy $p_{1, d}>p_{1, \min }$, $R_{1}(X)$ is the reliability index to satisfy $p_{1, d}<p_{1, \max }$, and $R_{i}(X)$ is the reliability index to satisfy $p_{i, d}<p_{i, \max }$. 
According to equations (4), (6), (12), and (13), the state function is as follows:

$$
\left\{\begin{array}{l}
g_{0}(X)=-\left(\frac{A}{\mu_{1}}\right)+N_{1} \cdot \Delta_{1}+N_{2} \cdot \Delta_{2}+\cdots+N_{i-1} \cdot \Delta_{i-1}+N_{i} \cdot \delta_{i}, \\
g_{1}(X)=-\left(N_{1} \cdot \Delta_{1}+N_{2} \cdot \Delta_{2}+\ldots+N_{i-1} \cdot \Delta_{i-1}+N_{i} \cdot \delta_{i}\right)+p_{1, \max }, \\
g_{2}(X)=-\left[\left(N_{1} \cdot \Delta_{1}+N_{2} \cdot \Delta_{2}+\ldots+N_{i-1} \cdot \Delta_{i-1}+N_{i} \cdot \delta_{i}\right) \times J_{2}+G_{2}\right]+p_{2, \max } \\
\ldots \\
g_{i}(X)=Q_{i}\left(\mu_{1}, \ldots, \mu_{i}, \Delta_{1}, \ldots, \Delta_{i-1}, \delta_{i}\right)+p_{i, \max },
\end{array}\right.
$$

where $Q_{i}$ is the relational expression between $\mu_{1}, \ldots, \mu_{i}$, $\Delta_{1}, \ldots, \Delta_{i-1}$, and $\delta_{i}$.

The reliability index $\beta_{i}$ corresponding to the state function $g_{i} \cdot(X)$ is expressed as

$$
\left\{\begin{array}{l}
\beta_{0}=\frac{\mu_{g_{0}}}{\sigma_{g_{0}}}=\frac{E\left[g_{0}(X)\right]}{\sqrt{\operatorname{Var}\left[g_{0}(X)\right]}} \\
\beta_{1}=\frac{\mu_{g_{1}}}{\sigma_{g_{1}}}=\frac{E\left[g_{1}(X)\right]}{\sqrt{\operatorname{Var}\left[g_{1}(X)\right]}} \\
\cdots \\
\beta_{i}=\frac{\mu_{g_{i}}}{\sigma_{g_{i}}}=\frac{E\left[g_{i}(X)\right]}{\sqrt{\operatorname{Var}\left[g_{i}(X)\right]}}
\end{array}\right.
$$

where $\mu_{g_{1}}, \ldots, \mu_{g_{i}}$ is the mean value of the state function and $\sigma_{g_{1}}, \ldots, \sigma_{g_{i}}$ is the standard deviation of the state function.

So, the reliability mathematical model of the multilayer interference fit can be obtained as

$$
R=\prod_{k=0}^{i+1} R_{i}
$$

where $R_{i}=\Phi\left(\beta_{i}\right), \Phi(\cdot)$ is a function conforming to the normal distribution. And normal distribution can be calculated by using function normcdf in MATLAB.

The wind turbine's shrink disk is the crucial connecting part between the shaft and gearbox. If it fails, the whole transmission system of the wind turbine will be completely invalid. Therefore, it requires a very high reliability. The shrink disk can be regarded as a three-layer interference fit model. This model consists of a shaft $\left(S_{1}\right)$, sleeve $\left(S_{2}\right)$, inner ring $\left(S_{3}\right)$, and outer ring $\left(S_{4}\right)$, as shown in Figure 2 . It forms the interference fit on the conical surface between the inner ring and outer ring by tightening the bolts, the cone angle of the conical surface is $\beta$, and the interference amount is $\delta_{3}$.
Because the interference between inner ring and outer ring causes compaction, the assembly clearance between inner ring and sleeve, and sleeve and shaft are counteracted to form the three-layer interference fit.

According to equation (16), the reliability mathematical model of the shrink disk is established as

$$
R_{s}=\prod_{k=0}^{4} R_{i} \text {. }
$$

A certain type of the shrink disk in Table 1 is selected as the application example to get the basic random variable $X$, including the friction coefficients $\mu_{1}, \mu_{2}$, and $\mu_{3}$ between shaft and sleeve, sleeve and inner ring, and inner ring and outer ring; the assembly clearance $\Delta_{1}$ and $\Delta_{2}$ between shaft and sleeve, and sleeve and inner ring; and the yield strength $\sigma_{S_{1}}, \ldots, \sigma_{S_{4}}$ of shaft, sleeve, inner ring, and outer ring. The mean and standard deviation are obtained by the empirical formula [16], and the results are shown in Table 2. In the design process, taking the interference amount $\delta_{3}$ between inner ring and outer ring as the primary design variable, the mean value of $\delta_{3}$ is given by design, and the standard deviation is based on the empirical formula with a value of $0.021 \mathrm{~mm}$.

In order to verify the accuracy of the reliability mathematical model, the result is verified by Monte Carlo simulation. Its reliability is equivalent to the product of probability:

$$
\begin{aligned}
R(X)= & P_{1}\left(p_{1, \min }(X)<p_{1, d}(X)<p_{1, \max }(X)\right) \\
& \times P_{2}\left(p_{2, d}(X)<p_{2, \max }(X)\right) \\
& \times P_{3}\left(p_{3, d}(X)<p_{3, \max }(X)\right) .
\end{aligned}
$$

In random variables $X_{1}, X_{2}, \ldots, X_{i}, \ldots, X_{N}, X_{i}$ satisfies the following inequalities:

$$
\left\{\begin{array}{l}
p_{1, \min }\left(X_{i}\right)<p_{1, d}\left(X_{i}\right)<p_{1, \max }\left(X_{i}\right), \\
p_{2, d}\left(X_{i}\right)<p_{2, \max }\left(X_{i}\right) \\
p_{3, d}\left(X_{i}\right)<p_{3, \max }\left(X_{i}\right) .
\end{array}\right.
$$




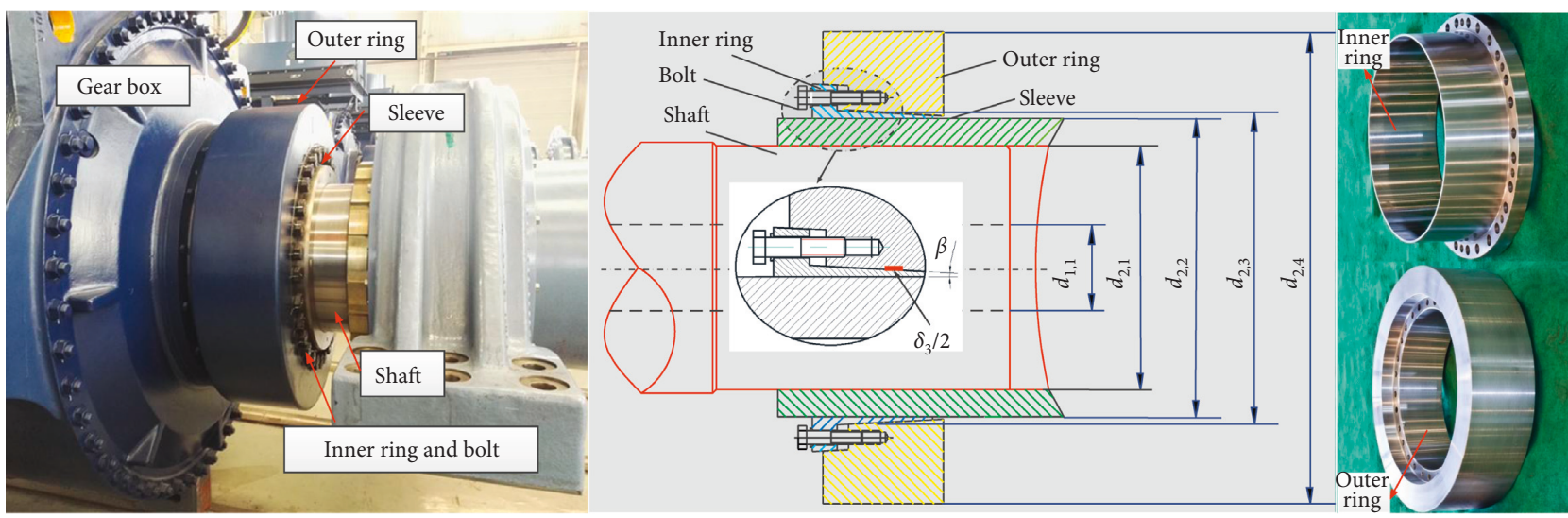

Figure 2: Schematic diagram of the wind turbine's shrink disk.

TABLE 1: The basic parameters of a certain type of the wind turbine's shrink disk.

\begin{tabular}{lc}
\hline Parameter & $\begin{array}{c}\text { Numerical } \\
\text { value }\end{array}$ \\
\hline $\begin{array}{l}\text { Inner diameter of shaft } d_{1,1}(\mathrm{~mm}) \\
\text { Outer diameter of shaft } d_{2,1}(\mathrm{~mm})\end{array}$ & 60 \\
Outer diameter of sleeve $d_{2,2}(\mathrm{~mm})$ & 640 \\
Average diameter of long conical surfaces of inner & 663.006 \\
ring $d_{2,3}(\mathrm{~mm})$ & 1020 \\
$\begin{array}{l}\text { Outer diameter of outer ring } d_{2,4}(\mathrm{~mm}) \\
\text { Length of contact surface between shaft and }\end{array}$ & 280.5 \\
sleeve $l_{1}(\mathrm{~mm})$ & \\
Elastic modulus of material for shaft, inner ring, and & 210 \\
outer ring $(\mathrm{GPa})$ & 180 \\
$\begin{array}{l}\text { Elastic modulus of material for sleeve (GPa) } \\
\text { Poisson's ratio }\end{array}$ & 0.3 \\
Assembly clearance between shaft and sleeve & 0.079 \\
Assembly clearance between sleeve and inner ring & 0.16 \\
\hline
\end{tabular}

TABLE 2: The value of random variable and standard deviation.

\begin{tabular}{lcc}
\hline $\begin{array}{l}\text { Random } \\
\text { variable }\end{array}$ & Mean value & Standard deviation \\
\hline$\mu_{1}$ & 0.15 & 0.02 \\
$\mu_{2}$ & 0.15 & 0.02 \\
$\mu_{3}$ & 0.045 & 0.006 \\
$R_{1}(\mathrm{~mm})$ & 0.079 & 0.019 \\
$R_{2}(\mathrm{~mm})$ & 0.16 & 0.027 \\
$\sigma_{S_{1}}(\mathrm{MPa})$ & 930 & 35.70 \\
$\sigma_{S_{2}}(\mathrm{MPa})$ & 835 & 30.03 \\
$\sigma_{S_{3}}(\mathrm{MPa})$ & 930 & 35.70 \\
$\sigma_{S_{4}}(\mathrm{MPa})$ & 930 & 35.70 \\
\hline
\end{tabular}

The number of random variables satisfying equation (19) is $U$, and the total number of random variables is $N, N=10^{4}$. According to the Monte Carlo method, the reliability problem is solved as [8]

$$
R_{M C S}=\frac{U}{N}
$$

Figure 3 shows the result contrast between reliability algorithm and Monte Carlo simulation. The results of both

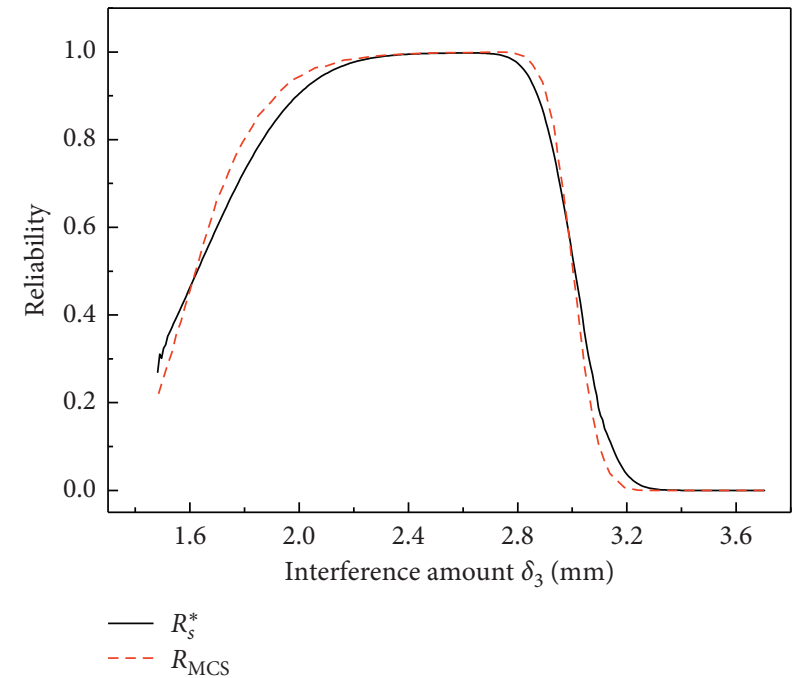

Figure 3: Reliability comparison of different methods.

methods have the same law and good coincidence. When $\delta_{3}$ is within $[1.5,2.2] \mathrm{mm}$, the reliability increases gradually, and the growth rate slows down gradually; when $\delta_{3}$ is within $[2.2,2.8] \mathrm{mm}$, the reliability basically remains unchanged; and when the $\delta_{3}$ is within $[2.8,3.2] \mathrm{mm}$, the reliability decreases rapidly. The initial increase in reliability is due to the increase in the interference amount to transmit more torque, and the final decrease in reliability is due to excessive interference amount, thus resulting in strength failure of the material. The recommended range of $\delta_{3}$ is $[2.2,2.8] \mathrm{mm}$.

\section{Multiobjective Intelligent Collaborative Design (MOICD)}

Based on the reliability mathematical model, the MOICD of the multilayer interference fit is proposed by introducing a lightweight design and considering the synergistic relationship between multiobjective and multivariable. Firstly, according to the design experience, the key variables of the multilayer interference fit design are determined, and the sample data of each key variable are obtained by the orthogonal experimental method [17]. Then, the Kriging 
method with good fitting effect on high-dimensional nonlinear problems is selected as a response surface approximation mathematical model to realize prediction [18]. And the approximation model can meet the prediction requirements of load transfer requirements, material strength, and equipment weight.

Among them, considering the stress concentration problem, equivalent stress is regarded as the indicator of material strength. Next, NSGA-II is selected as the optimal algorithm to realize MOICD of the multilayer interference fit, comparing the algorithm of NSGA-II [19], multiobjective simulated annealing (MOSA), and multiobjective particle swarm optimization (MOPSO). Figure 4 shows the flow chart of the MOICD method. $d_{i}$ is the combined diameter of cylinders $S_{i}$ and $S_{i+1}$.

The MIOCD of the multilayer interference fit is implemented by taking the wind turbine's shrink disk as an application example. The design indicators corresponding to the approximation model is determined, including the minimum contact pressure $p_{1, \text { min }}$ between shaft and sleeve, the maximum equivalent contact stress $E_{\max }$, and the minimum mass $m_{\min }$. Then, related indicators are converted to optimized size parameters [16], including the minimum diameter $d_{3 \text { min }}$ of long cone surface of the inner ring (consistent with $d_{3 \min }=d_{2,3}-b$, where $d_{2,3}$ is the average diameter of long conical surfaces of the inner ring and $b$ is a coefficient related to size), the diameter $d_{2,4}$ of the outer ring, and the maximum design interference amount $\delta_{3 \max }$ between inner ring and outer ring; the relationship between these size parameters is shown in Figure 2.

Using the orthogonal experimental method, the corresponding result data of design indicators and size parameters are determined as shown in Table 3, and the relevant results are obtained from finite element ABAQUS simulation. The value range of $d_{3 \min }$ and $d_{2,4}$ is given by combining with the production process of the shrink disk. The recommended range of the interference amount for the reliability mathematical model is $[2.2,2.8] \mathrm{mm}$; in order to get more data points, on this basis, extend the scope of $\delta_{3 \max }$ to $[2,3] \mathrm{mm}$.

An approximate response surface mathematical model based on design indicators (objective function) and size parameters is constructed by the Kriging method, as shown in equations (21) (23), and this approximate model is achieved by using DACE toolbox of MATLAB:

$$
\begin{aligned}
f_{1}\left(d_{2,4}, \delta_{3 \max }, d_{3 \min }\right)= & 107120.0208+6.417 d_{2,4} \\
& +2058.9388 \delta_{3 \max }-339.6408 d_{3 \min } \\
& -0.001205 d_{2,4}^{2}-3.5228 \delta_{3 \max }^{2} \\
& +0.2652 d_{3 \min }^{2}+0.004408 d_{2,4} \delta_{3 \max } \\
& -2.5722 \delta_{3 \max } d_{3 \min } \\
& -0.005774 d_{2,4} d_{3 \min }+\varepsilon_{1},
\end{aligned}
$$

$$
\begin{aligned}
f_{2}\left(d_{2,4}, \delta_{3 \max }, d_{3 \min }\right)= & -1781.9778+0.1413 d_{2,4} \\
& +153.5583 \delta_{3 \max }+5.2007 d_{3 \min } \\
& -0.0002795 d_{2,4}^{2}+0.2560 \delta_{3 \max }^{2} \\
& -0.004458 d_{3 \min }^{2}+0.1090 d_{2,4} \delta_{3 \max } \\
& -0.2918 \delta_{3 \max } d_{3 \min } \\
& +0.0006041 d_{2,4} d_{3 \min }+\varepsilon_{2},
\end{aligned}
$$

$$
f_{3}\left(d_{2,4}, \delta_{3 \max }, d_{3 \min }\right)=-666.6891+0.001627 d_{2,4}^{2}+\varepsilon_{3},
$$

where $f_{1}\left(d_{2,4}, \delta_{3 \max }, d_{3 \min }\right)$ is the maximum equivalent stress function, $f_{2}\left(d_{2,4}, \delta_{3 \max }, d_{3 \min }\right)$ is the function of minimum contact pressure between shaft and sleeve, $f_{3}\left(d_{2,4}, \delta_{3 \max }, d_{3 \min }\right)$ is the minimum mass function, $\varepsilon_{i} \quad(i=1,2,3)$ is residual, the expectation is zero, and the covariance is not zero.

The coefficient of determination $R^{2}$ is used to test the accuracy of the Kriging method, and its expression is as follows:

$$
R^{2}=\frac{\sum_{i=1}^{h}\left(\hat{y}_{i}-\bar{y}\right)^{2}}{\sum_{i=1}^{h}\left(y_{i}-\bar{y}\right)^{2}}
$$

where $h$ is the number of data sample points, $\hat{y}_{i}$ is the predicted value of the approximate model, $y_{i}$ is the true value, and $\bar{y}$ is the average value. The larger the decision coefficient $R^{2}$ is in the range of $[0,1]$, the higher the accuracy of the model. The correlation coefficient of $f_{1}\left(d_{4}, \delta_{3 \max }\right.$, $\left.d_{3 \min }\right), \ldots, f_{3}\left(d_{4}, \delta_{3 \max }, d_{3 \min }\right)$ is $0.999954,0.999999$, and 1. It shows that the Kriging method meets the accuracy requirement.

According to the main causes of the interference fit failure such as insufficient load transfer and the maximum equivalent stress exceeding the material yield limit [7], the multiobjective model of the shrink disk first meets the requirements of torque and yield strength, and then meets the minimum mass. The mathematical expression of the multiobjective model is as follows:

$$
\begin{array}{ll}
\min & F(x)=\left[-f_{1}(x),-f_{2}(x), f_{3}(x)\right], \\
\text { s.t. } & E_{\max }<Q, \\
& p_{1, \min }>\frac{2 M_{t}}{\pi \mu_{1} l_{1} d_{1}}, \\
& d_{4 \min } \leq d_{2,4} \leq d_{4 \max }, \\
& \delta_{3 \max \min } \leq \delta_{3 \max } \leq \delta_{3 \max \max }, \\
& d_{3 \min \min } \leq d_{3 \min } \leq d_{3 \min \max } .
\end{array}
$$




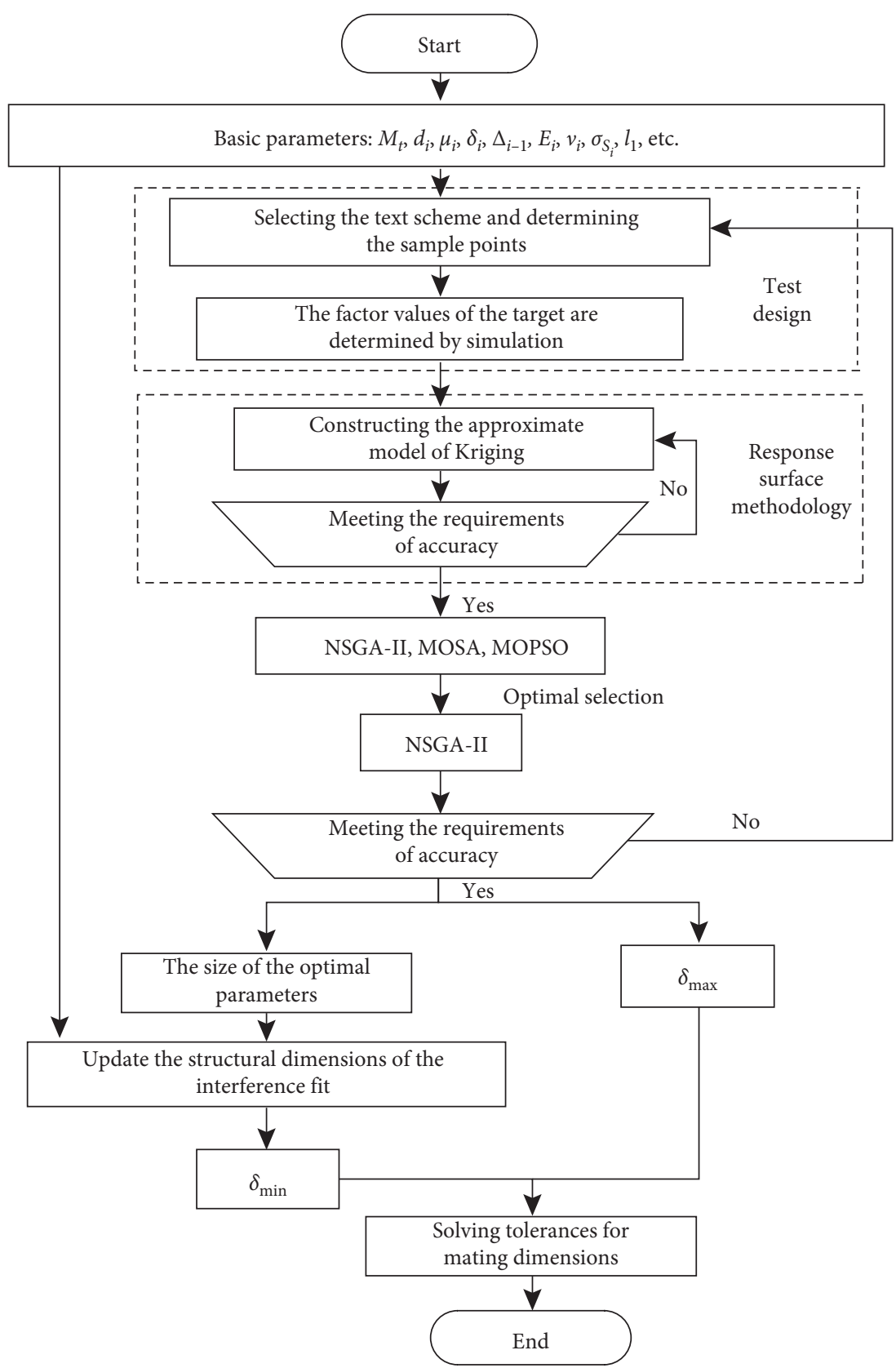

FIGURE 4: Flow chart of the MOICD method.

TABLE 3: Data obtained by the orthogonal experimental method.

\begin{tabular}{lcccccc}
\hline Sample points & $d_{2,4}(\mathrm{~mm})$ & $\delta_{3 \max }(\mathrm{mm})$ & $d_{3 \min }(\mathrm{mm})$ & $E_{\max }(\mathrm{MPa})$ & $p_{1, \min }(\mathrm{MPa})$ & $m_{\min }(\mathrm{kg})$ \\
\hline 1 & 1000 & 2 & 648 & 657.019 & 117.279 & 960.970 \\
2 & 1000 & 2 & 652 & 638.443 & 114.912 & 960.970 \\
3 & 1000 & 2 & 656 & 622.318 & 112.473 & 960.970 \\
4 & 1000 & 2.5 & 648 & 850.578 & 154.505 & 960.970 \\
5 & 1000 & 2.5 & 652 & 820.793 & 151.672 & 960.970 \\
6 & 1000 & 2.5 & 656 & 802.022 & 148.636 & 960.970 \\
7 & 1000 & 3 & 648 & 1039.589 & 191.921 \\
8 & 1000 & 3 & 652 & 1003.183 & 188.497 & 960.970 \\
9 & 1000 & 3 & 656 & 980.801 & 184.872 & 960.970 \\
10 & 1020 & 2 & 648 & 664.741 & 120.915 & 1026.727 \\
11 & 1020 & 2 & 652 & 641.577 & 118.686 & 1026.727 \\
\hline
\end{tabular}


TABle 3: Continued.

\begin{tabular}{|c|c|c|c|c|c|c|}
\hline Sample points & $d_{2,4}(\mathrm{~mm})$ & $\boldsymbol{\delta}_{3 \max }(\mathrm{mm})$ & $d_{3 \min }(\mathrm{mm})$ & $E_{\max }(\mathrm{MPa})$ & $p_{1, \min }(\mathrm{MPa})$ & $m_{\min }(\mathrm{kg})$ \\
\hline 12 & 1020 & 2 & 656 & 625.665 & 116.272 & 1026.727 \\
\hline 13 & 1020 & 2.5 & 648 & 854.312 & 159.347 & 1026.727 \\
\hline 14 & 1020 & 2.5 & 652 & 826.184 & 156.546 & 1026.727 \\
\hline 15 & 1020 & 2.5 & 656 & 804.984 & 153.539 & 1026.727 \\
\hline 16 & 1020 & 3 & 648 & 1044.206 & 197.857 & 1026.727 \\
\hline 17 & 1020 & 3 & 652 & 1009.640 & 194.471 & 1026.727 \\
\hline 18 & 1020 & 3 & 656 & 984.447 & 190.882 & 1026.727 \\
\hline 19 & 1040 & 2 & 648 & 668.134 & 124.451 & 1093.787 \\
\hline 20 & 1040 & 2 & 652 & 644.916 & 122.243 & 1093.787 \\
\hline 21 & 1040 & 2 & 656 & 628.195 & 119.969 & 1093.787 \\
\hline 22 & 1040 & 2.5 & 648 & 860.380 & 163.912 & 1093.787 \\
\hline 23 & 1040 & 2.5 & 652 & 829.261 & 161.141 & 1093.787 \\
\hline 24 & 1040 & 2.5 & 656 & 809.633 & 158.162 & 1093.787 \\
\hline 25 & 1040 & 3 & 648 & 1045.534 & 203.590 & 1093.787 \\
\hline 26 & 1040 & 3 & 652 & 1013.402 & 200.104 & 1093.787 \\
\hline 27 & 1040 & 3 & 656 & 988.631 & 196.678 & 1093.787 \\
\hline
\end{tabular}

The maximum equivalent stress $E_{\max }$ cannot exceed the material yield limit $Q$, the minimum contact pressure $p_{1 \text {,min }}$ must be greater than the pressure required to transmit the rated torque, and $d_{3 \text { min }}, d_{2,4}$, and $\delta_{3 \text { max }}$ meet the upper and lower limit requirements. According to design experience $[9,10], E_{\max }$ and $p_{1, \min }$ need to be as large as possible; that is, the negative number of its objective function $-f_{1}(x)$ and $-f_{2}(x)$ need to be as small as possible, and at the same time, $f_{3}(x)$ is as small as possible to reduce the mass of the shrink disk and lower the manufacturing cost. The priority of the above three functions is as follows: $-f_{2}(x)>-f_{1}(x)>f_{3}(x)$.

Equations (21) (25) are solved using NSGA-II, MOSA, and MOPSO algorithm. Among these three algorithms, NSGA-II setting: the population number is 1000 , the variation rate is 0.1 , and the crossing rate is 0.85 ; MOSA setting: the Boltzmann coefficient is 1 , the maximum internal loop number is 1000 , and the cooling coefficient is 0.99 ; MOPSO setting: the population number is 1000 and the nearest population is 2 . The certain type of the shrink disk is designated as an example, and the three algorithms are used to run 20 times independently [20]. The minimum mass obtained by these three algorithms is the same $f_{3}(x)=960.970$. For a more intuitive comparison of the three algorithms, $-f_{1}$ $(x)$ and $-f_{2}(x)$ are taken as the research objects, and the contrast is shown in Figure 5.

Figure 5 shows the optimal solution sets of $-f_{1}(x)$ and $-f_{2}(x)$. The results revealed that NSGA-II has significant advantages over other algorithms in optimal performances [20]. Specifically, the negative number of maximum equivalent stress and minimum contact pressure from NSGA-II is smaller than that from MOSA and MOPSO. That is to say, the maximum equivalent stress and minimum contact pressure obtained by NSGA-II are the largest; besides, the computational optimization time required for NSGA-II is less than that for the other two algorithms, as shown in Table 4 . Table 4 is a comparison of the average computational time required by the three algorithms when calculating the results in Figure 5. Finally, NSGA-II is selected as the optimal algorithm.
In the calculation result of NSGA-II, the minimum value of $-f_{1}(x)$ and $-f_{2}(x)$ is chosen as the best solution of optimal solution sets. Then, $d_{3 \text { min }}$ and $d_{2,4}$ are combined to update the overall structure size. Simultaneously in equations (1) and (6), the minimum design interference amount $\delta_{3 \mathrm{~min}}$ between inner ring and outer ring is as follows:

$$
\begin{gathered}
\delta_{3 \text { min }}=-K_{3,3} p_{2 \text { min }}+\left(K_{1,4}+K_{4,3}\right) p_{3 \text { min }}, \\
p_{2 \text { min }}=\frac{R_{1}+\left(K_{1,2}+K_{4,1}\right) p_{1 \text { min }}}{K_{2,2}} \\
p_{3 \text { min }}=\frac{R_{2}-K_{3,2} p_{1 \text { min }}+\left(K_{4,2}+K_{1,3}\right) p_{2 \text { min }}}{K_{2,3}} .
\end{gathered}
$$

According to equation (7), the minimum contact pressure $p_{1 \mathrm{~min}}$ is obtained, where $p_{2 \text { min }}$ is the minimum contact pressure between sleeve and inner ring and $p_{3 \text { min }}$ is the minimum contact pressure between inner ring and outer ring. $K_{i j}$ is the relational expression for the known parameters of the shrink disk.

The optimized size parameters $d_{3 \mathrm{~min}}, d_{2,4}, \delta_{3 \mathrm{~min}}$, and $\delta_{3 \text { max }}$ are determined by the MOICD method and compared with the dimension data obtained by the traditional design method, as shown in Table 5. The traditional design method combines equations (4) and (12) to achieve the design of main dimensions by calculating contact pressure, without considering the problems of stress concentration and lightweight [21].

As can be seen from Table $5, d_{2,4}$ of the MOICD method is less than that of the traditional method, which reduces the mass of the shrink disk by $6.7 \%$. With $\delta_{3 \text { min }}$ as the input parameter, the minimum contact pressure $p_{1 \text { min }}$ is obtained by using equation (4), which proves that the rated torque $M_{t}$ designed by the MOICD method is improved compared with the traditional method. With $\delta_{3 \max }$ as the input parameter, the results of the two methods are compared using finite element ABAQUS analysis. The feasibility of the finite element model is verified by the stress test experiment. The 


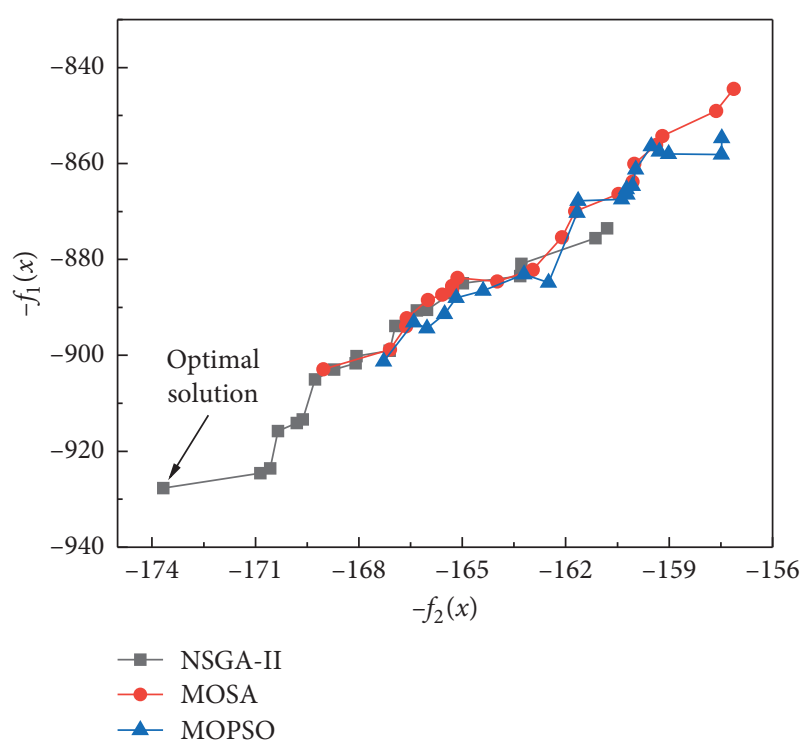

FIGURE 5: Comparison of the optimal solution sets of $-f_{1}(x)$ and $-f_{2}$ $(x)$ in three algorithms.

TABLe 4: The mean time use of three algorithms.

\begin{tabular}{lccc}
\hline Time & NSGA-II & $\begin{array}{c}\text { Algorithm } \\
\text { MOSA }\end{array}$ & MOPSO \\
\hline $\begin{array}{l}\text { Mean time of } \\
\text { use (s) }\end{array}$ & 15.07 & 44.07 & 22.31 \\
\hline
\end{tabular}

TABLE 5: Comparison of design results between the traditional and MOICD methods.

\begin{tabular}{lcc}
\hline $\begin{array}{l}\text { Design } \\
\text { results }\end{array}$ & $\begin{array}{c}\text { Traditional } \\
\text { method }\end{array}$ & $\begin{array}{c}\text { MOICD } \\
\text { method }\end{array}$ \\
\hline$d_{2,4}(\mathrm{~mm})$ & 1020 & 1000 \\
$d_{3 \min }(\mathrm{mm})$ & 652 & 653.8 \\
$\delta_{3 \min }(\mathrm{mm})$ & 1.841 & 1.907 \\
$\delta_{3 \max }(\mathrm{mm})$ & 3.310 & 2.820 \\
$m_{\min }(\mathrm{kg})$ & 1026.727 & 960.970 \\
$M_{t}(\mathrm{kN} \cdot \mathrm{m})$ & 2800.2 & 2800.7 \\
\hline
\end{tabular}

test method is selected to fit the triaxial strain rosette. The schematic diagram of the experiment is shown in Figure 6. In the experimental scheme, two CS-1 dynamic resistance strain gauges and one XHCDSP data acquisition instrument are used. The experimental stress value is the average value of four strain rosette tests, and the error with the finite element simulation is $2.31 \%$.

The modeling data of the finite element are shown in Table 1 . The friction coefficient of the contact surface between the shaft and sleeve, and the sleeve and inner ring is set to 0.15 , and that between the inner ring and outer ring (coated with molybdenum disulfide grease) is 0.045 . This finite element model belongs to the problem of the multicontact surface with tangential slip, so the contact mode is selected as general contact, the contact surface is defined by the penalty function friction formula, and the contact pair is used as the finite sliding of surface-to-surface contact.
Considering the structural symmetry of the shrink disk, in order to reduce the calculation cost, the two-dimensional axisymmetric reduced integral element CAX4R is selected as the finite element model cell type $[22,23]$. The total number of units is 7828. The mesh of the model is divided into a quadrilateral, and the mesh sizes of the outer ring, inner ring, sleeve, and shaft are $4 \mathrm{~mm}, 2 \mathrm{~mm}, 4 \mathrm{~mm}$, and $4 \mathrm{~mm}$, respectively [9]. The layout seeds are determined by the length of edges, which optimizes the aspect ratio of the mesh and improves the mesh quality. The average aspect ratio of the shaft, sleeve, inner ring, and outer ring meshes is $1.02,1$, 1.41, and 1.03, respectively [24]. Setting the boundary conditions, the shaft $U X=U Y=0$, the sleeve $U X=U Y=0$, the inner ring $U X=0, U Y=\delta_{3} / 2 \sin \beta \cos \beta$, and the outer ring is no boundary conditions. The type of analysis step is set to static general. Because the assembly process is a large displacement problem, the geometric nonlinearity is set to On. At the same time, the incremental step is set to a fixed value, and the maximum incremental step is defined as 0.02 . Figure 7 is the stress distribution diagram of the finite element model of the traditional method and MOICD method.

Figure 7 shows that the maximum equivalent stress of the shrink disk based on the traditional method is $1103.646 \mathrm{MPa}$, which is much higher than the maximum yield strength $930 \mathrm{MPa}$, and plastic deformation occurs. According to the conventional design theory [21], plastic deformation has a negative impact on the strength of mechanical structures, resulting in a reduction in service life. The maximum equivalent stress based on the MOICD method is $915.313 \mathrm{MPa}$, which is less than the yield limit of the material. Comprehensive analysis proves that the shrink disk based on the MOICD method has been improved in the design objectives of minimum mass, torque transfer, and yield strength, which meets the multiobjective design requirements.

\section{Multiparameter Analysis of Multilayer Interference Fit}

4.1. The Influence of Outermost Diameter of Enveloping Part on the Contact Pressure. Multiparameter analysis of the multilayer interference fit is carried out using finite element software ABAQUS. Figures 8 10 show the impact of the outermost diameter of the enveloping part (outer ring diameter $d_{2,4}$ ) and axial contact position on contact pressure of the shrink disk. $d_{2,4}$ takes $1000 \mathrm{~mm}, 1020 \mathrm{~mm}$, and $1040 \mathrm{~mm}$ as research objects.

In Figures 8 10, the contact pressure of each interference layer increases with $d_{2,4}$, and the change of contact pressure from the innermost interference layer between shaft and sleeve to the outermost interference layer between inner ring and outer ring decreases continuously. $d_{2,4}$ is smaller due to the lower contact pressure, which could not meet the torque transmission requirements. A larger $d_{2,4}$ is conducive to increase the contact pressure and help the shrink disk to undertake more torque, but it will increase the mass and manufacturing cost. Therefore, the multiobjective optimization of $d_{2,4}$ can not only meet 

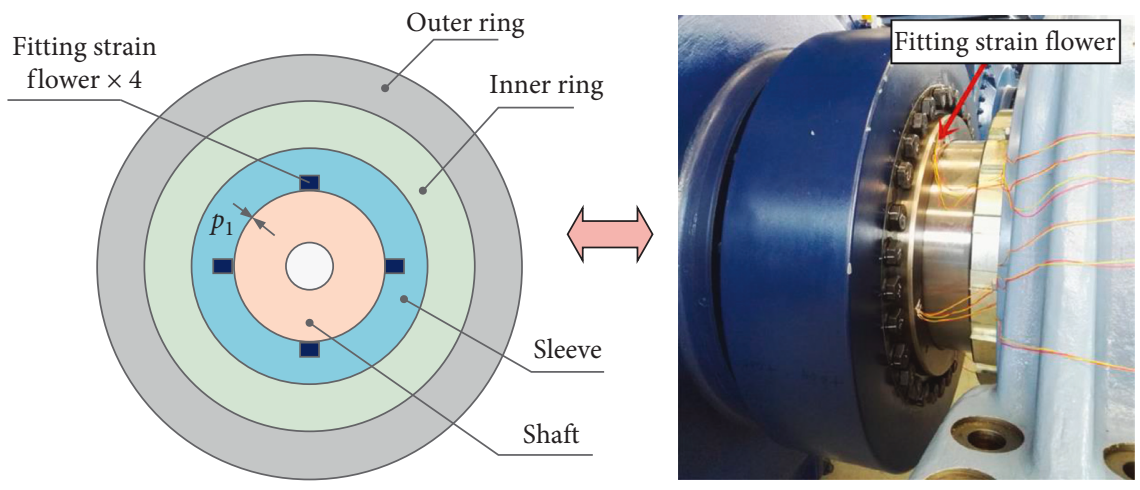

FIgURE 6: The schematic diagram of the experiment.

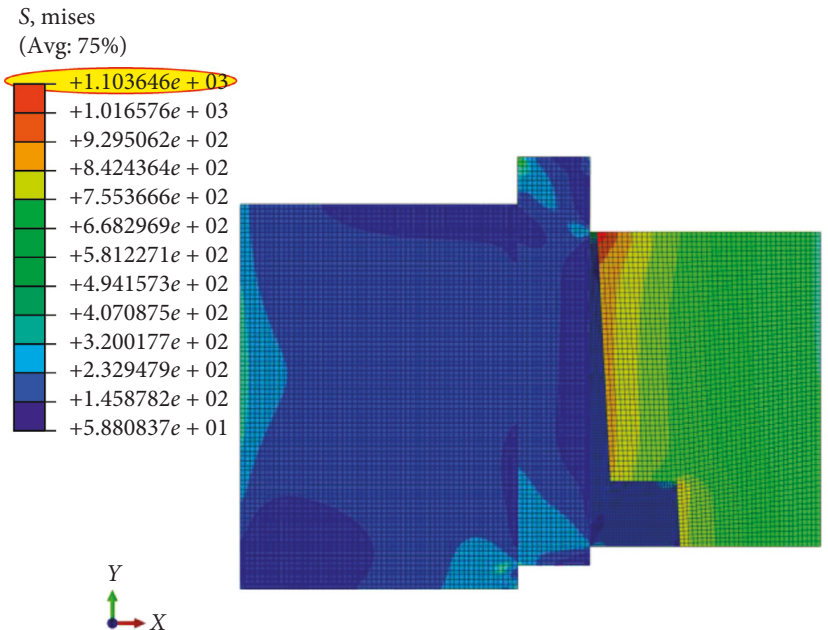

(a)

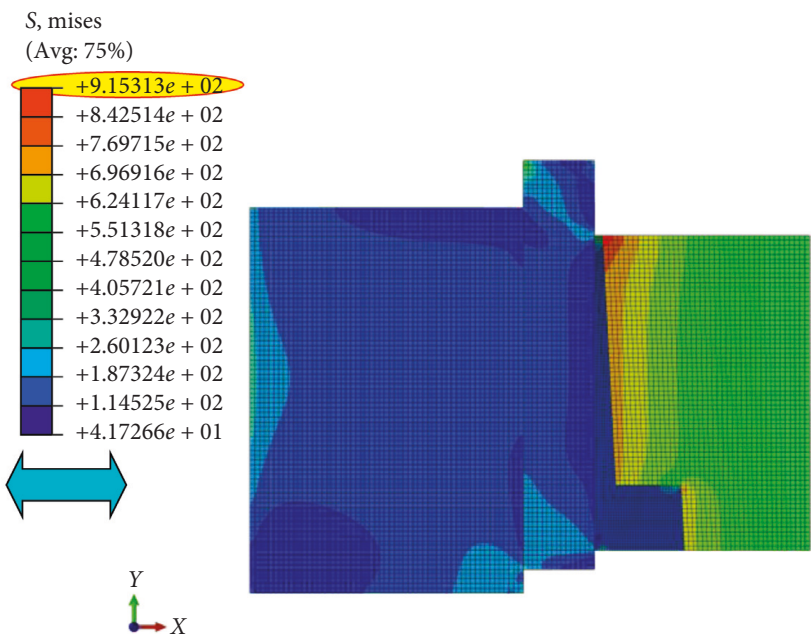

(b)

Figure 7: Stress distribution diagram based on two methods.

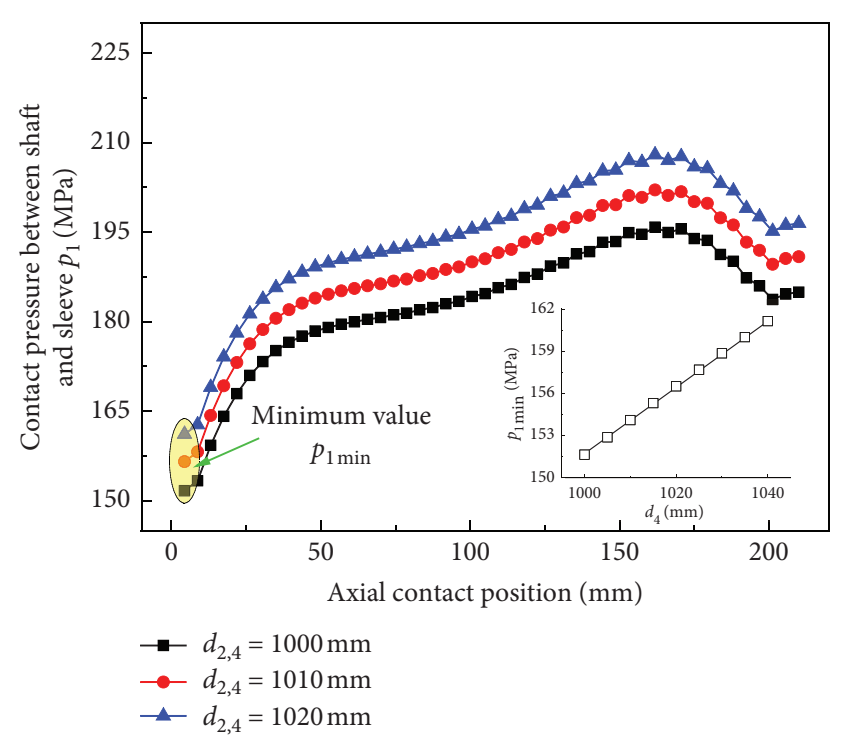

Figure 8: The influence of $d_{2,4}$ and axial contact position on $p_{1}$.

the load transfer requirements but also help the shrink disk to reduce the mass and achieve the design goal of lightweight.
4.2. The Influence of Subouter Diameter of Enveloping Part on Contact Pressure. Figures 11 13 show the influence of suboutermost diameter of enveloping part (the minimum diameter $d_{3 \text { min }}$ in the inner ring long cone) and axial contact position on contact pressure of the shrink disk. $d_{3 \text { min }}$ takes $648 \mathrm{~mm}, 652 \mathrm{~mm}$, and $656 \mathrm{~mm}$ as research objects.

In Figures 11 13, the contact pressure of each interference layer decreases with $d_{3 \mathrm{~min}}$, and the change of contact pressure from the innermost interference layer to the outermost interference layer decreases continuously. In addition, the pressure distribution of the contact layer between inner ring and outer ring is also affected by $d_{3 \text { min. }}$ A smaller $d_{3 \text { min }}$ could increase the contact pressure and help the shrink disk transmit more load, but it will increase the difficulty of machining inner ring. Therefore, in the design process of the inner ring, smaller $d_{3 \text { min }}$ should be selected as the design size based on considering appropriate processing technology.

4.3. The Influence of Interference Amount on Contact Pressure. Figures 14 16 show the impact of interference amount $\delta_{3}$ and axial contact position on contact pressure of the shrink disk. $\delta_{3}$ takes $2 \mathrm{~mm}, 2.5 \mathrm{~mm}$, and $3 \mathrm{~mm}$ as research objects, 


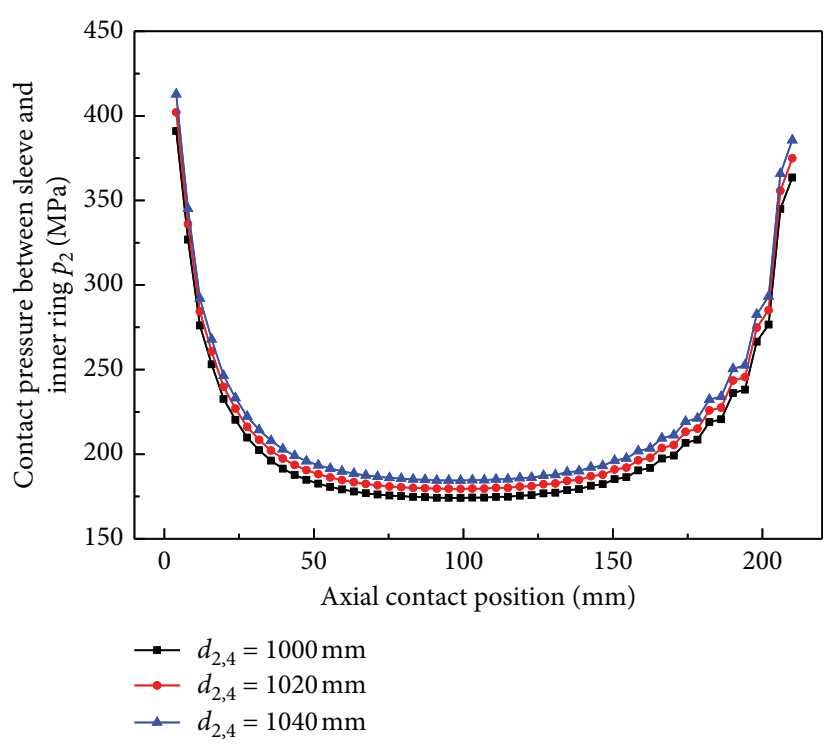

Figure 9: The influence of $d_{2,4}$ and axial contact position on $p_{2}$.

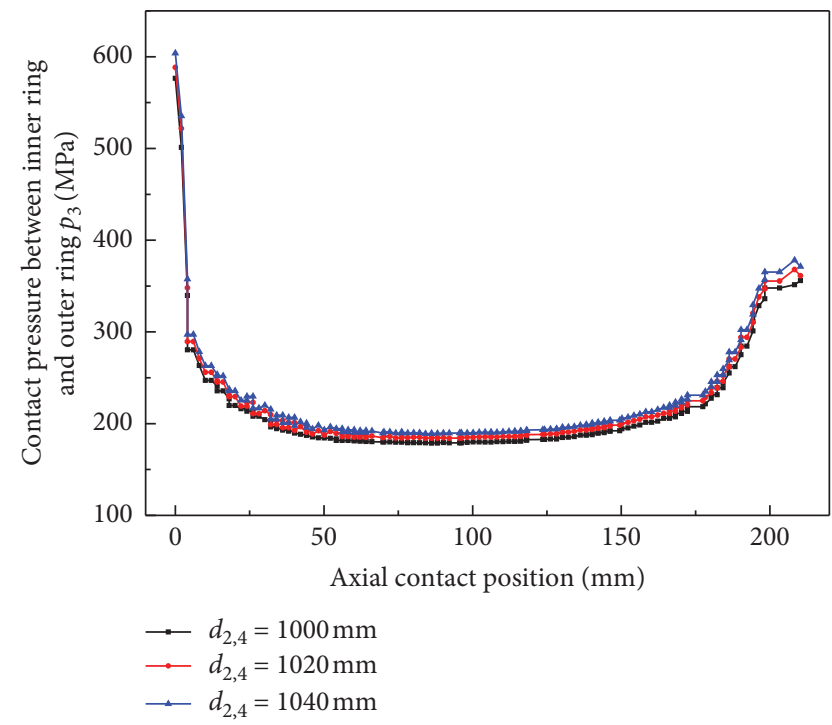

FIgURE 10: The influence of $d_{2,4}$ and axial contact position on $p_{3}$.

and there is a positive correlation between $\delta_{3}$ and contact pressure; the change of contact pressure from the innermost interference layer to outermost interference layer is decreasing. Too small $\delta_{3}$ design results in insufficient contact pressure which could not transfer rated torque, and excessive $\delta_{3}$ design results in excessive contact pressure which is straightforward to produce plastic deformation; therefore, it is necessary to strictly control the interference amount.

The rules shown in Figures 8 16 verify the correctness of equations (4) and (6). In addition, these figures show that there is a large contact pressure singularity at both ends of the interference layer, and the singularity of the contact surface between inner ring and outer ring is the largest, which is caused by the stress concentration of the interference fit. Stress concentration is often the leading cause of damage, so it must be considered in the design.

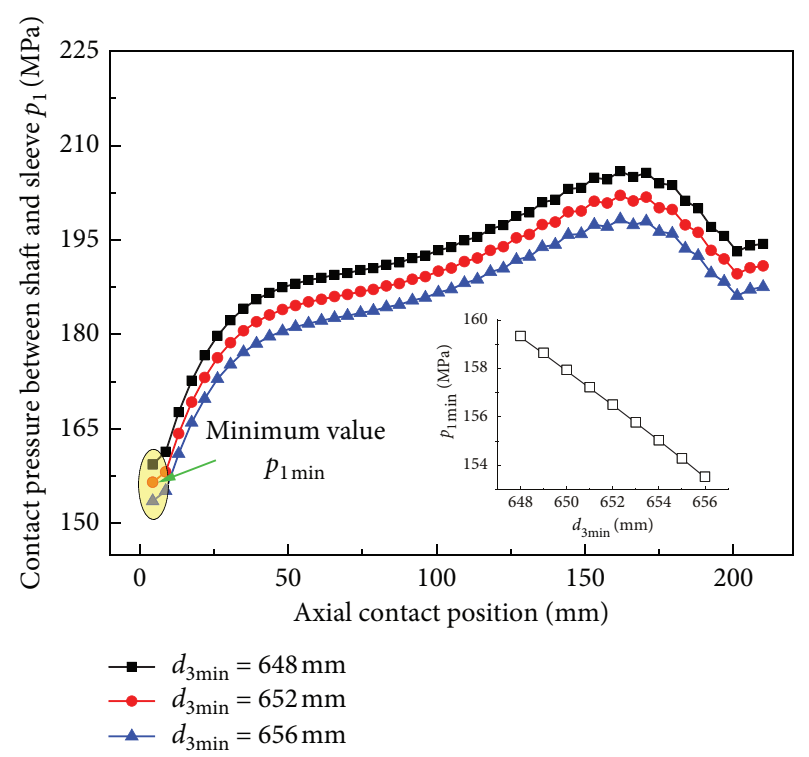

Figure 11: The influence of $d_{3 \text { min }}$ and axial contact position on $p_{1}$.

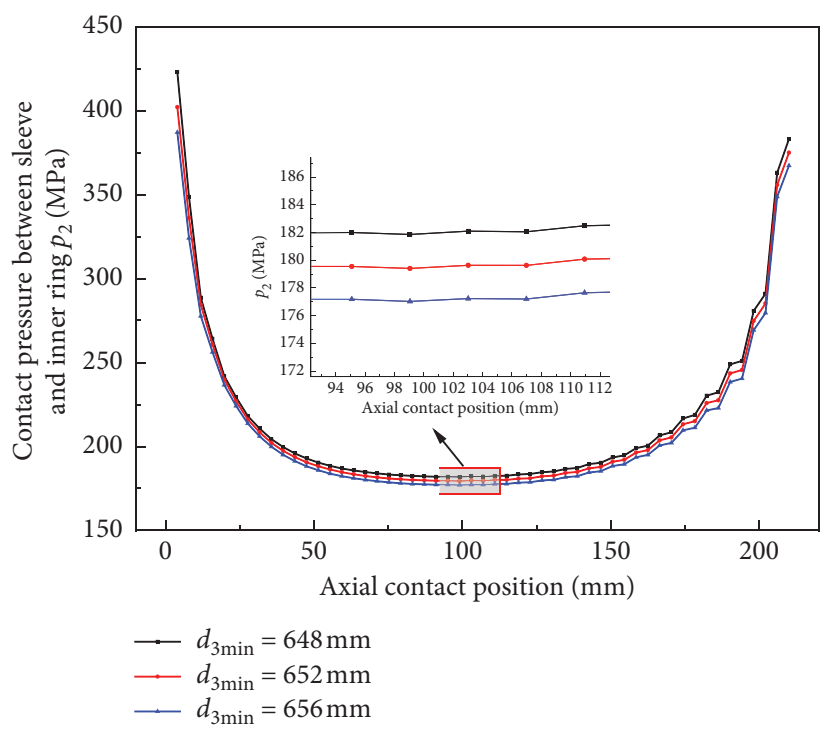

FIgURE 12: The influence of $d_{3 \text { min }}$ and axial contact position on $p_{2}$.

\section{Test Verification}

In order to test whether the optimized wind turbine's shrink disk meets the requirements of the application, based on the optimized design size in Table 5, shrink disk samples are manufactured, and the experimental verification is completed by a specific test bench. This test bench exerts torque using a hydraulic cylinder and supporting bracket, as shown in Figure 17. According to experimental experience, the torque retention time is 3 minutes. It is determined that no circumferential slip occurs in the three interference fit layers of the sample, which proves to meet the requirements of torque design. The test data are shown in Table 6, $M=2800 \mathrm{kN} \cdot \mathrm{m}$, which is illustrated in Table 1 . 


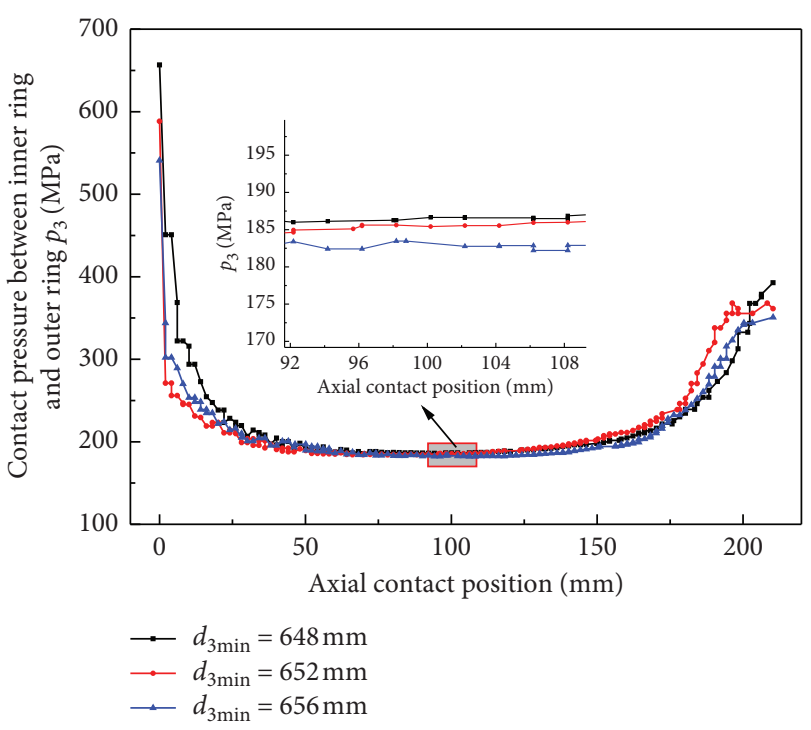

FIGURE 13: The influence of $d_{3 \min }$ and axial contact position on $p_{3}$.

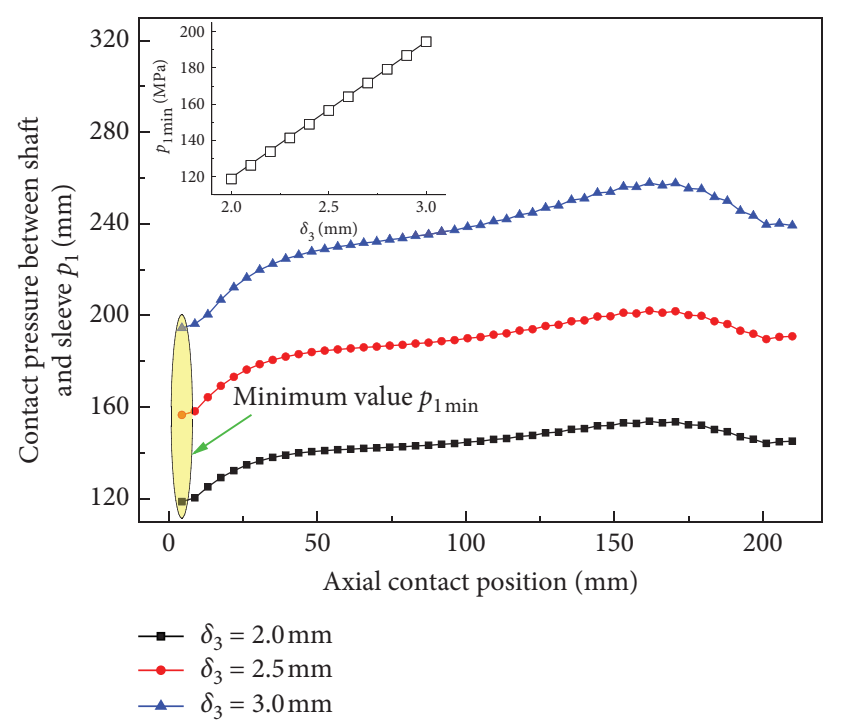

Figure 14: The influence of $\delta_{3}$ and axial contact position on $p_{1}$.

\section{Conclusions}

(i) The mechanical model and the relationship of contact pressure among each interference layer are obtained, the reliability mathematical model on the reliability evaluation method is built, and the range of recommended interference amount is given.

(ii) Considering lightweight design and stress concentration, the MOICD method for the multilayer interference fit is proposed. Taking the Kriging model as the prediction method of the response surface, comparing with NSGA-II, MOSA, and MOPSO, NSGA-II is proved to be the optimal algorithm for multilayer interference fit design.

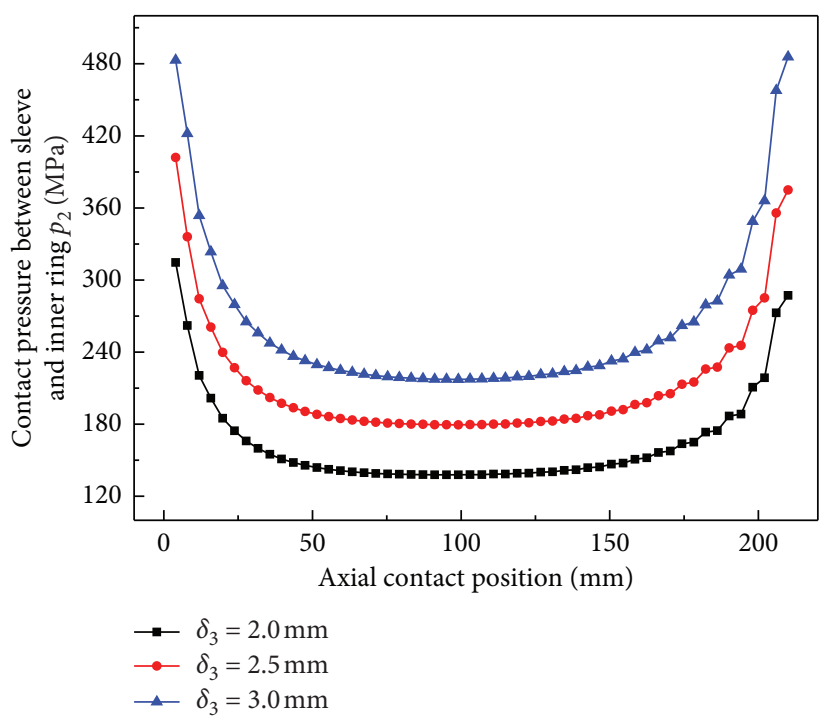

FIgURE 15: The influence of $\delta_{3}$ and axial contact position on $p_{2}$.

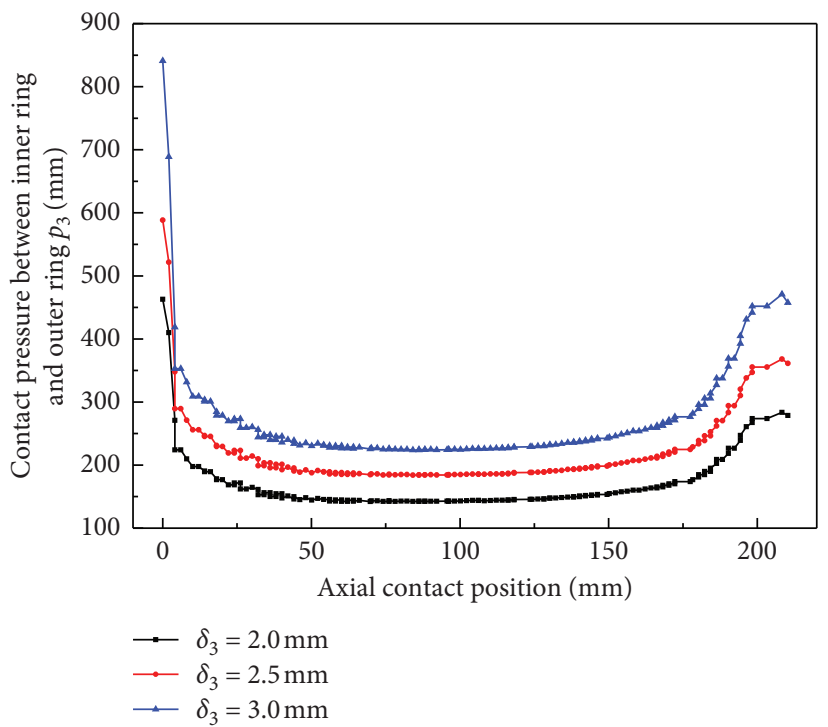

Figure 16: The influence of $\delta_{3}$ and axial contact position on $p_{3}$.

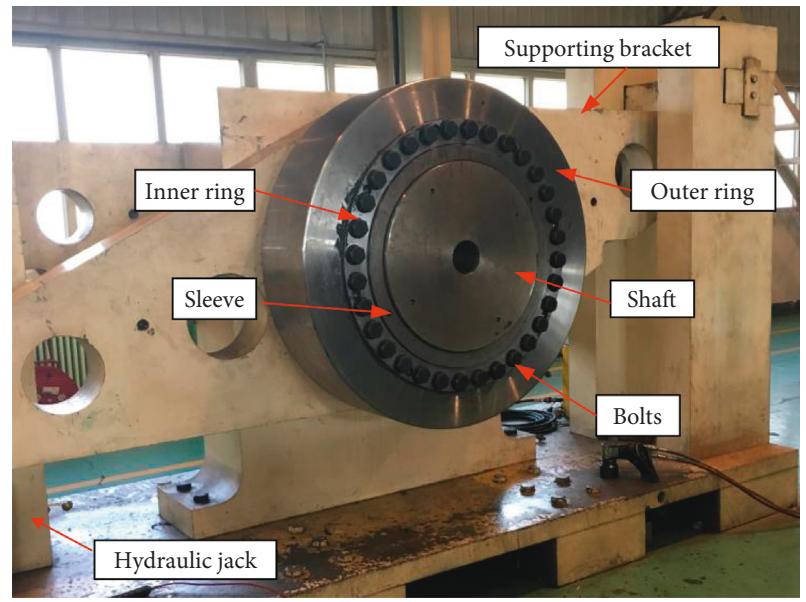

Figure 17: Test bench of the wind turbine's shrink disk. 
TABle 6: Static torque detection data.

\begin{tabular}{lcc}
\hline $\begin{array}{l}\text { Torque } \\
\text { value }(\mathrm{kN} \cdot \mathrm{m})\end{array}$ & $\begin{array}{c}\text { Circumferential } \\
\text { slip }(\mathrm{mm})\end{array}$ & $\begin{array}{c}\text { Holding } \\
\text { pressure time }(\mathrm{min})\end{array}$ \\
\hline $0.4 M$ & 0 & 3 \\
$0.5 M$ & 0 & 3 \\
$0.65 M$ & 0 & 3 \\
$0.8 M$ & 0 & 3 \\
$M$ & 0 & 3 \\
$1.2 M$ & 0 & 3 \\
\hline
\end{tabular}

(iii) Using the equivalent stress as the evaluation index of the stress concentration problem, the design defects due to stress concentration in traditional design can be avoided [7]. Comparing finite element analysis and mechanical calculation, the maximum equivalent stress on the shrink disk is less than the yield strength of the material, and at the same time, the transmission torque is increased and the weight is reduced, ultimately the optimization goal of the MOICD method is achieved.

(iv) The rules of different parameters on contact pressure are obtained using multiparameter analysis on outermost layer diameter, subouter layer diameter, and interference amount, which guides the design and manufacturing optimization of the multilayer interference fit.

(v) According to the design sizes from the MOICD method, the sample is developed, and the torque is exerted gradually on the shaft by the test platform. Experiments show that the shrink disk can meet the requirements of torque design.

\section{Data Availability}

The data used to support the findings of this study are included within the article.

\section{Conflicts of Interest}

The authors declare that there are no conflicts of interest regarding the publication of this article.

\section{Acknowledgments}

This research was supported by the National Natural Science Foundation of China (No. 51875382), Shanxi Provincial Key Research and Development Plan (No. 201803D421103), Shanxi Provincial Key Science and Technology Special Projects (No. 20181102023), Postgraduate Innovation Project of Shanxi Province (2018SY089), and the Coordinative Creation Center of Taiyuan Heavy Machinery Equipment (1331 Project).

\section{References}

[1] A. Strozzi, E. Bertocchi, A. Baldini, and S. Mantovani, "Normalization of the stress concentrations at the rounded edges of an interference fit between a solid shaft subjected to bending and a hub," Mechanics Based Design of Structures and Machines, vol. 44, no. 4, pp. 405-425, 2016.

[2] Y. Sun, W. Hu, F. Shen, Q. Meng, and Y. Xu, "Numerical simulations of the fatigue damage evolution at a fastener hole treated by cold expansion or with interference fit pin," International Journal of Mechanical Sciences, vol. 107, pp. 188-200, 2016.

[3] T. Wu, K. Zhang, H. Cheng, P. Liu, D. Song, and Y. Li, "Analytical modeling for stress distribution around interference fit holes on pinned composite plates under tensile load," Composites Part B: Engineering, vol. 100, pp. 176-185, 2016.

[4] C. Zeng, W. Tian, and W. H. Liao, "The effect of residual stress due to interference fit on the fatigue behavior of a fastener hole with edge cracks," Engineering Failure Analysis, vol. 66, pp. 72-87, 2016.

[5] A. Bahloul, M. M. Mhalla, and C. Bouraoui, "Crack repair of SENT specimen using the interference fit process," Journal of Alloys and Compounds, vol. 748, pp. 363-374, 2018.

[6] P. Zou, Y. Li, K. Zhang, P. Liu, and H. Zhong, "Mode I delamination mechanism analysis on CFRP interference-fit during the installation process," Materials \& Design, vol. 116, pp. 268-277, 2017.

[7] X. Wang, Z. Lou, X. Wang, X. Hao, and Y. Wang, "Prediction of stress distribution in press-fit process of interference fit with a new theoretical model," Proceedings of the Institution of Mechanical Engineers, Part C: Journal of Mechanical Engineering Science, vol. 233, no. 8, pp. 2834-2846, 2019.

[8] J. Wang, K. Ning, J. Xu, and Z. Li, "Reliability-based robust design of wind turbine's shrink disk," Proceedings of the Institution of Mechanical Engineers, Part C: Journal of Mechanical Engineering Science, vol. 232, no. 15, pp. 2685-2696, 2018.

[9] J. M. Wang, K. Ning, L. Tang, R. Malekian, Y. Liang, and Z. Li, "Modeling and finite element analysis of load-carrying performance of a wind turbine considering the influence of assembly factors," Applied Sciences, vol. 7, no. 3, p. 298, 2017.

[10] J. M. Wang, J. F. Kang, and L. Tang, "Theoretical and experimental studies for wind turbine's shrink disk," Proceedings of the Institution of Mechanical Engineers, Part C: Journal of Mechanical Engineering Science, vol. 229, no. 2, pp. 325-334, 2014.

[11] J. Qiu and M. Zhou, "Analytical solution for interference fit for multi-layer thick-walled cylinders and the application in crankshaft bearing design," Applied Sciences, vol. 6, no. 6, p. 167, 2016.

[12] Z. Bai, J. Wang, K. Ning, and D. Hou, "Contact pressure algorithm of multi-layer interference fit considering centrifugal force and temperature gradient," Applied Sciences, vol. 8, no. 5, p. 726, 2018.

[13] L. You, K. Yan, T. Shi, J. Man, and N. Liu, "Analytical solution for the effect of anisotropic layers/interlayers on an elastic multi-layered medium subjected to moving load," International Journal of Solids and Structures, vol. 172-173, pp. 10-20, 2019.

[14] L. You, K. Yan, Y. Hu, and W. Ma, "Impact of interlayer on the anisotropic multi-layered medium overlaying viscoelastic layer under axisymmetric loading," Applied Mathematical Modelling, vol. 61, pp. 726-743, 2018.

[15] J. M. Wang, D. F. Tao, Q. X. Huang et al., “Algorithm research on contact pressure and magnitude of interference for multilayer cylinder's interference fit," Engineering Mechanics, vol. 30, pp. 270-275, 2013. 
[16] J. M. Wang and L. Tang, Research on Theory and Method of Shrink, Metallurgical Industry Press, Beijing, China, 2014.

[17] Y. Wang and X. Huo, "Multiobjective optimization design and performance prediction of centrifugal pump based on orthogonal test," Advances in Materials Science and Engineering, vol. 2018, Article ID 6218178, 10 pages, 2018.

[18] M. Xiao, L. Gao, X. Shao, H. Qiu, and P. Jiang, "A generalised collaborative optimisation method and its combination with kriging metamodels for engineering design," Journal of Engineering Design, vol. 23, no. 5, pp. 379-399, 2012.

[19] M. D. Shieh, Y. Li, and C. C. Yang, "Product form design model based on multiobjective optimization and multicriteria decision-making," Mathematical Problems in Engineering, vol. 2017, Article ID 5187521, 15 pages, 2017.

[20] N. Chau, T. Dao, and V. Nguyen, "An efficient hybrid approach of finite element method, artificial neural networkbased multiobjective genetic algorithm for computational optimization of a linear compliant mechanism of nanoindentation tester," Mathematical Problems in Engineering, vol. 2018, Article ID 7070868, 19 pages, 2018.

[21] D. X. Cheng, Handbook of mechanical design, connection and fastening, Vol. 1, Chemical Industry Press, Beijing, China, 2004.

[22] R. Lostado, R. F. Martinez, and B. J. Mac Donald, "Determination of the contact stresses in double-row tapered roller bearings using the finite element method, experimental analysis and analytical models," Journal of Mechanical Science and Technology, vol. 29, no. 11, pp. 4645-4656, 2015.

[23] R. Lostado, R. E. García, and R. F. Martinez, "Optimization of operating conditions for a double-row tapered roller bearing," International Journal of Mechanics and Materials in Design, vol. 12, no. 3, pp. 353-373, 2016.

[24] R. F. Martinez, R. L. Lorza, A. A. S. Delgado, and N. O. P. Pullaguari, "Optimizing presetting attributes by softcomputing techniques to improve tapered roller bearings working conditions," Advances in Engineering Software, vol. 123, pp. 13-24, 2018. 


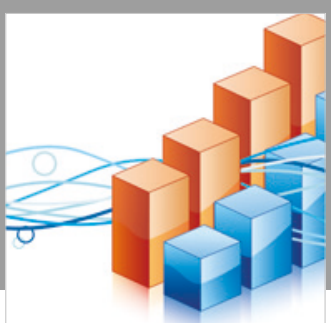

Advances in

Operations Research

\section{-n-m}
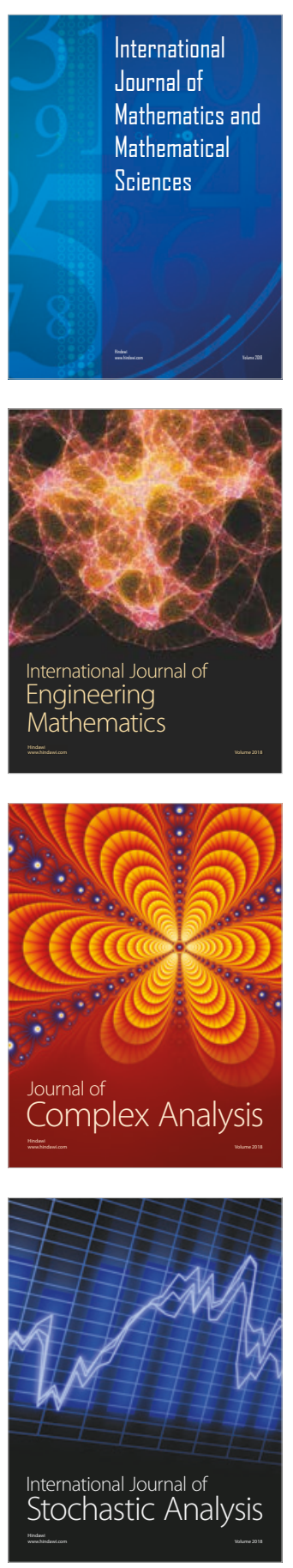
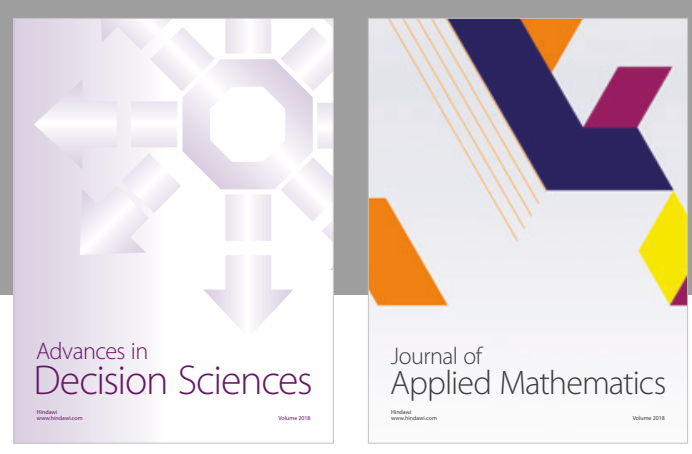

Journal of

Applied Mathematics
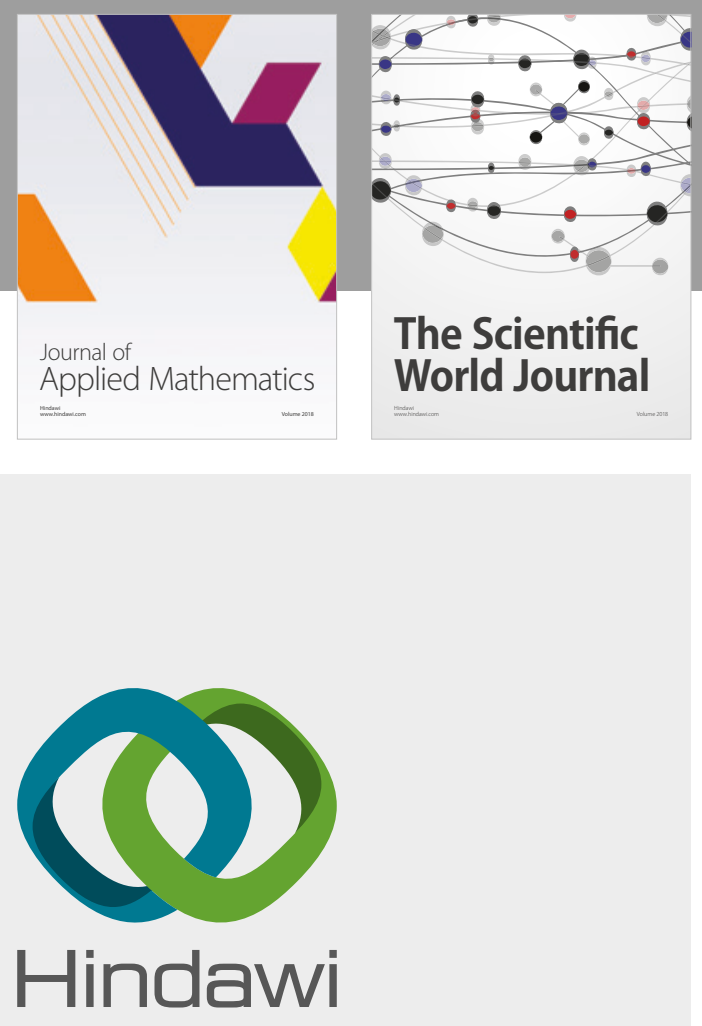

Submit your manuscripts at

www.hindawi.com

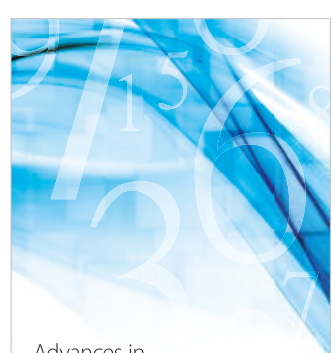

Advances in
Numerical Analysis
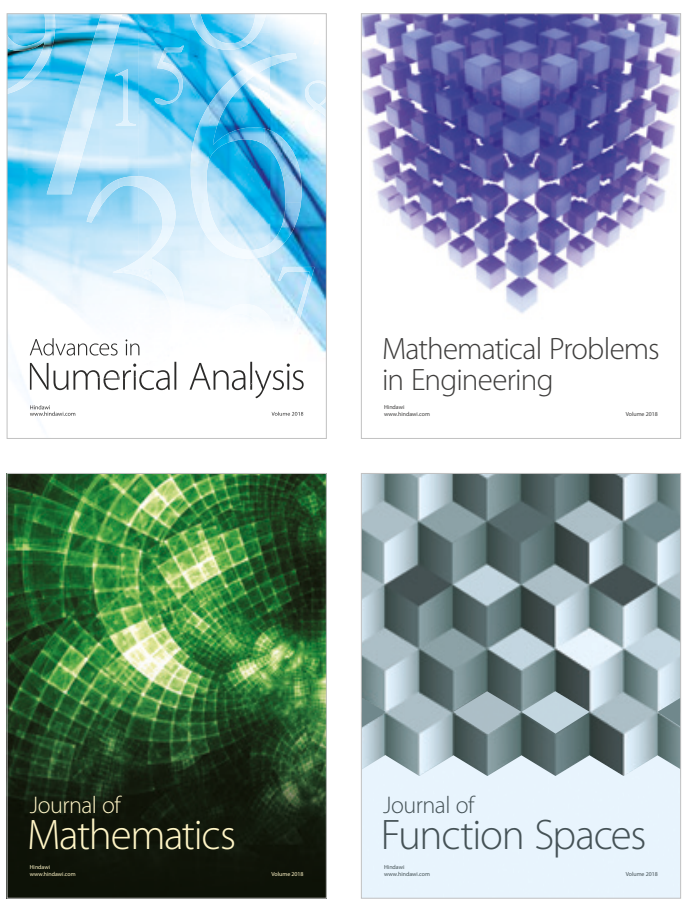

Mathematical Problems in Engineering

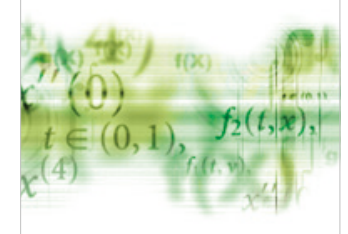

International Journal of

Differential Equations

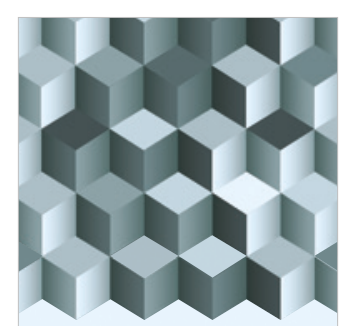

Journal of

Function Spaces

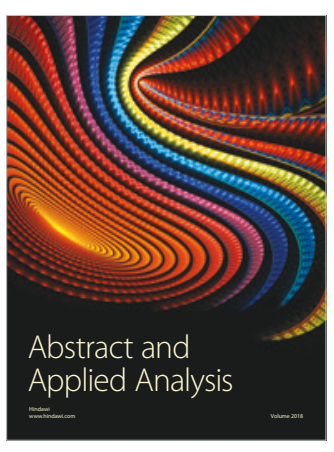

The Scientific

World Journal

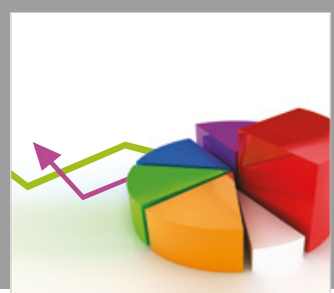

Journal of

Probability and Statistics
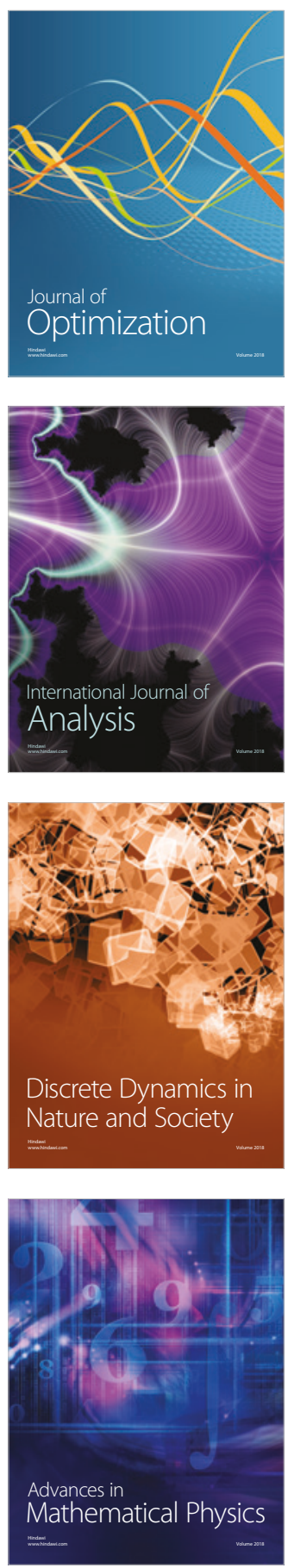